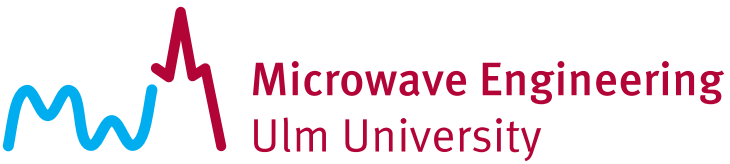

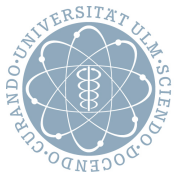

\section{Automotive Radar Interference Mitigation by Reconstruction and Cancellation of Interference Component}

Jonathan Bechter and Christian Waldschmidt

(c) 2015 IEEE. Personal use of this material is permitted. Permission from IEEE must be obtained for all other uses, in any current or future media, including reprinting/republishing this material for advertising or promotional purposes, creating new collective works, for resale or redistribution to servers or lists, or reuse of any copyrighted component of this work in other works. 


\title{
Automotive Radar Interference Mitigation by Reconstruction and Cancellation of Interference Component
}

\author{
Jonathan Bechter and Christian Waldschmidt \\ Institute of Microwave Techniques, University of Ulm, Germany \\ Email: jonathan.bechter@uni-ulm.de
}

\begin{abstract}
Automotive radar interference is an issue arising with the increasing amount of radar systems in automotive scenarios. Interference can influence the functionality of a radar system, e.g. decrease its detection capability. To ensure functionality in critical situations, interference countermeasures are applied. This paper proposes a countermeasure for chirp sequence modulated radars interfering one another estimating an interfering signals' parameters to afterwards reconstruct and remove the interference.
\end{abstract}

\section{INTRODUCTION}

When considering radar as a standard component for future generations of automobiles, scenarios with multiple users radiating and receiving $\mathrm{RF}$ signals in similar frequency bands will be a common issue. As power from one radar system is transmitted into the receiving structure of another, the other radar might get altered in its functionality. The radar generating the disturbing power is called interferer, the other one is called victim. For the investigation of radar interference and its mitigation, analytical considerations have been performed. Problems arising with radar interference have been investigated taking account of modulation schemes of victim and interferer. This behaviour was investigated for interference between pulsed and FMCW radars [1],[2],[3],[4]. It was shown that interference between two FMCW systems may lead to serious limitations. If the frequency ramps from victim and interferer cross each other as shown in Fig. 1, the noise floor will increase significantly [2],[4], so low power targets might not be detected anymore. If parallel frequency ramps of interferer and victim occur, this could lead to the appearence of ghost targets in the receiver structure. However, as there is no correlation between the phase noise of victim and interferer, these targets would show up as very broad peaks in the spectrum [4].

To counteract interference, multiple procedures are possible. Methods were proposed scanning the received time domain signal to remove corrupted samples [2], mitigating the interference in space domain [5], or by shifting the transmit frequency in frequency domain [6]. There is a plenty of possible other ideas, but not all are working for each radar modulation. On the following pages, an interference countermeasure in time domain suited for chirp sequence modulated radars interfered by chirp sequence or FMCW modulation schemes is presented.

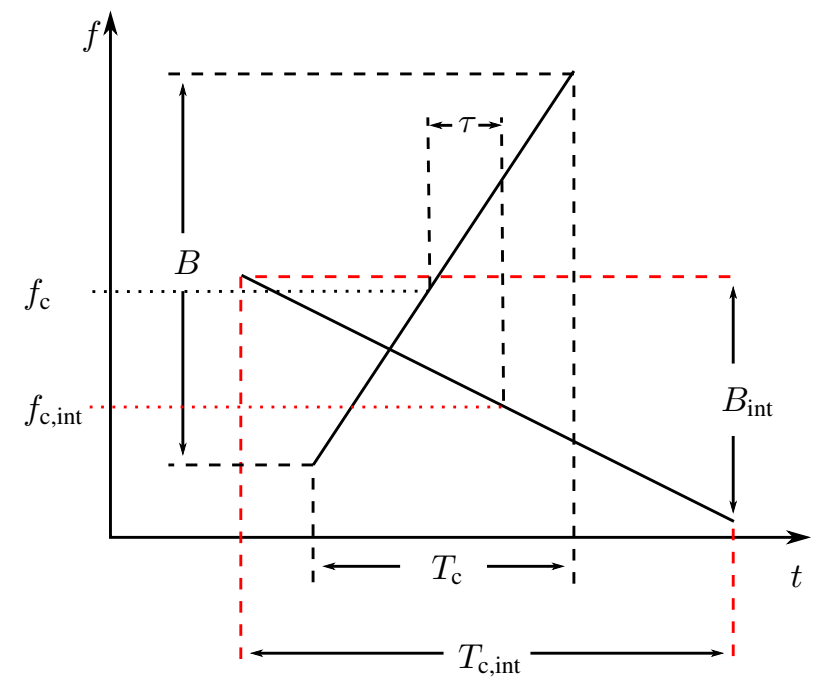

Fig. 1: Two frequency ramps cutting each other. The line with index 'int' represents the interfering radar, the other one the victim. The variables are describing chirp duration, bandwidth, and center frequency of both signals. $\tau$ is a time shift between the starting points of both ramps.

\section{THEORETICAL INVESTIGATION}

Considering non parallel frequency ramps as shown in Fig. 1, interference only occurs as long as an interfering signal passes the victim radar's receiver bandwidth. Therefore, in time domain interference will only show up for a limited period,what can be seen in Fig. 2. It can be seen that the amplitude level of the interfering signal is higher than the target signals' amplitudes. This is expected, as the interfering signal need not be reflected by a target before reaching the receiving antenna [2]. In frequency domain, interference as illustrated leads to an increase of the noise floor and masking of low power targets.

As mentioned above, one countermeasure in time domain is to detect whether a sample or a period of samples is affected by interference and afterwards replacing those samples by empty values. By doing so, the interference is completely removed from the signal and cannot increase the noise floor anymore. However, this method has the disadvantage that a part of the signal is lost, leaving a hole in the measured data set. This lacking data will be the more problematic the longer 


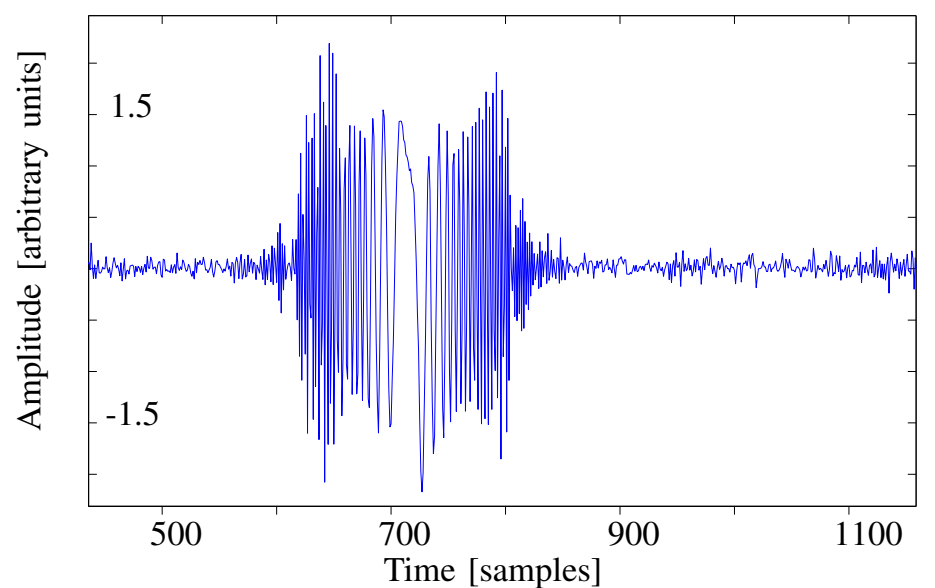

Fig. 2: Time domain signal of a single chirp of a chirp sequence modulated radar interfered by a chirp sequence modulated radar. Interference occurs around the samples 620 to 820 .

the interference period lasts. In order not to lose information this way, the method for interference mitigation proposed here is to estimate the parameters of the interfering signal based on the received time domain samples. With the parameters known, the interfering signal component can be reconstructed and removed from the total received signal without any loss of information on target signals. Therefore, an analytical derivation is performed.

The frequency $f_{\mathrm{Tx}}(t)$ sent by a chirp sequence modulated radar transmitter can be described as

$$
f_{\mathrm{Tx}}(t)=f_{\mathrm{c}}+\frac{B}{T_{\mathrm{c}}} t,
$$

where $f_{\mathrm{c}}$ is the carrier frequency, $B$ the bandwidth and $T_{\mathrm{c}}$ the duration of a single chirp [7]. A similar description can be done for the frequency $f_{\text {int }}(t)$ of an interfering signal sent by a radar system also using a chirp sequence modulation:

$$
f_{\text {int }}(t)=f_{\mathrm{c}, \text { int }}+\frac{B_{\text {int }}}{T_{\mathrm{c}, \text { int }}}(t-\tau) .
$$

The variables describe the same relations as in (1), with the enhanced indices related to an interfering signal. $\tau$ represents a time shift between the starting points of the interfering signal's frequency ramp and the interfered radar's frequency ramp. These frequency ramps are illustrated in Fig. 1 for two signals with different parameters.

In a chirp sequence modulated radar, downconversion of one chirp of a reflected signal from the $i$-th target leads to the baseband signal [7]:

$$
s_{\text {target }, i}=A_{i} \cos \left(2 \pi\left[\frac{2 f_{\mathrm{c}} R_{i}}{c}+\left(\frac{2 f_{\mathrm{c}} v_{i}}{c}+\frac{2 B R_{i}}{c T_{\mathrm{c}}}\right) t\right]\right)
$$

with $A_{i}$ the signal amplitude, $R_{i}$ and $v_{i}$ the target's range and velocity, and $c$ the speed of light. For the further considerations, it is suitable to summarize the items into some constants to

$$
s_{\text {target }, i}(t)=A_{i} \cos \left(2 \pi f_{i} t+\varphi_{i}\right) \text {. }
$$

It can be seen that the signal received from target $i$ is an oscillation with constant amplitude and frequency. If one interfering signal $s_{\text {int }}(t)$ is present, the total received signal $s_{\mathrm{Rx}}(t)$ is the sum of all target responses (4) and the interference:

$$
\begin{aligned}
s_{\mathrm{Rx}}(t) & =\sum_{i} s_{\text {target }, i}(t)+s_{\text {int }}(t) \\
& =\sum_{i} A_{i} \cos \left(2 \pi f_{i} t+\varphi_{i}\right)+s_{\text {int }}(t) .
\end{aligned}
$$

The time domain interference signal can be determined by mixing of the two frequency ramps in Fig. 1. When the receiver lowpass filter is assumed ideal, this will result in a cosine term with constant amplitude. So during the interference period, the interference component can generally be described as

$$
s_{\text {int }}(t)=A_{\text {int }} \cos \left(\varphi_{\text {int }}(t)\right) \text {. }
$$

The interferer amplitude $A_{\text {int }}$ is constant as for a target response, but its phase $\varphi_{\text {int }}(t)$ will not have the same linear behaviour as a target signal phase. The calculation of $\varphi_{\text {int }}(t)$ is done in a similar way as the derivation of the target signal phase in [7]. Using (1) and (2), mixing of the transmitted chirp signal with the received interference signal will provide the baseband interference phase $\varphi_{\text {int }}(t)$ :

$$
\begin{aligned}
\varphi_{\text {int }}(t)= & 2 \pi \int f_{\text {int }}(t) d t-2 \pi \int f_{\mathrm{Tx}}(t) d t \\
= & 2 \pi \int\left(f_{\text {int }}(t)-f_{\mathrm{Tx}}(t)\right) d t \\
= & 2 \pi \int\left(f_{\mathrm{c}, \text { int }}-f_{\mathrm{c}}\right. \\
& \left.+\left(\frac{B_{\text {int }}}{T_{\mathrm{c}, \text { int }}}-\frac{B}{T_{\mathrm{c}}}\right) t-\frac{B_{\text {int }}}{T_{\mathrm{c}, \text { int }}} \tau\right) d t
\end{aligned}
$$

In the following steps, the phase $\varphi_{\text {int }}(t)$ and the amplitude $A_{\text {int }}$ will be determined to afterwards remove the interference.

To estimate the phase response, the derivative of the received signal $s_{\mathrm{Rx}}(t)$ is calculated. Derivation of (5) leads to

$$
\begin{aligned}
\frac{d}{d t} s_{\mathrm{Rx}}(t)= & \frac{d}{d t} s_{\text {int }}(t)+\frac{d}{d t} \sum_{i} s_{\text {target }, i}(t) \\
= & -A_{\text {int }} \sin \left(\varphi_{\text {int }}(t)\right) \frac{d}{d t} \varphi_{\text {int }}(t) \\
& +\sum_{i}\left(-2 \pi f_{i} A_{i} \sin \left(2 \pi f_{i} t+\varphi_{i}\right)\right) .
\end{aligned}
$$

As mentioned before, the interfering signal has a much higher amplitude as the targets' reflections. So (8) is approximated by

$$
\frac{d}{d t} s_{\mathrm{Rx}}(t) \approx-A_{\mathrm{int}} \sin \left(\varphi_{\mathrm{int}}(t)\right) \frac{d}{d t} \varphi_{\mathrm{int}}(t) .
$$

In Fig. 3, a simulated time domain signal is shown, in Fig. 4 its derivative. It can be seen that the interference component is dominating the signal derivative, so the approximation is assumed valid. Taking a look at $\frac{d}{d t} \varphi_{\text {int }}(t)$, the integral in (7) will disappear when calculating its derivation:

$$
\frac{d}{d t} \varphi_{\mathrm{int}}(t)=2 \pi\left(f_{\mathrm{c}, \text { int }}-f_{\mathrm{c}}+\left(\frac{B_{\mathrm{int}}}{T_{\mathrm{c}, \text { int }}}-\frac{B}{T_{\mathrm{c}}}\right) t-\frac{B_{\mathrm{int}}}{T_{\mathrm{c}, \text { int }}} \tau\right) .
$$




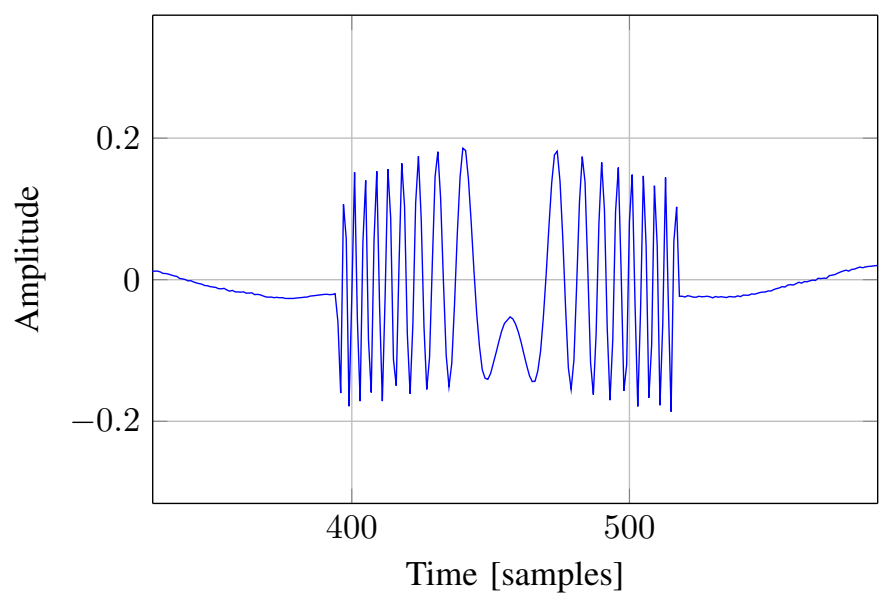

Fig. 3: Simulated time domain signal of one chirp of a chirp sequence radar with an interferer crossing through the receiver bandwidth leading to a time limited interference.

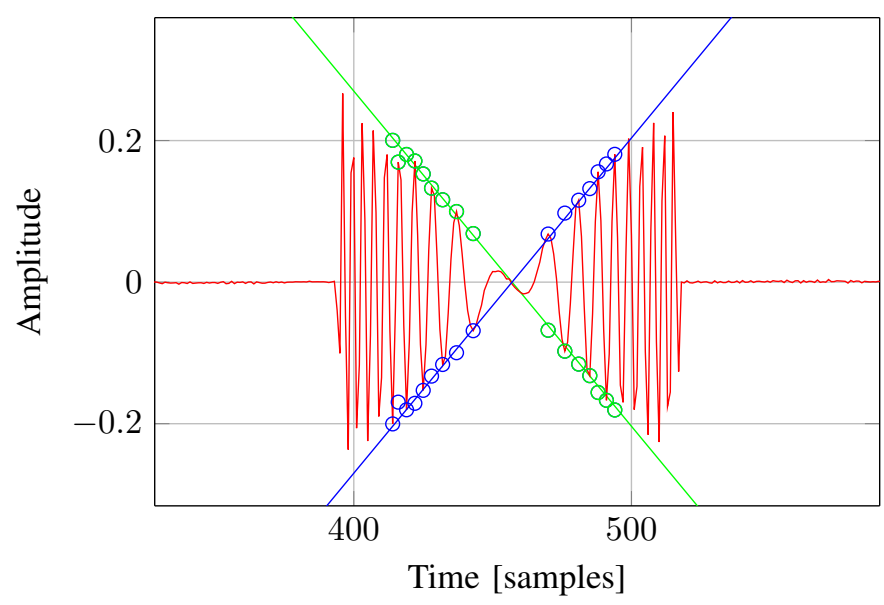

Fig. 4: Derivative of Fig. 3 in red. With the extreme values, a straight line can be fitted holding the phase behaviour of the interferer. There are two possible solutions, just different in a factor -1 .

By introducing constants $c_{1}$ and $c_{2}$, this can be considered as a linear equation of the form

$$
\frac{d}{d t} \varphi_{\text {int }}(t)=c_{1} \cdot t+c_{2}
$$

When the sine in (9) reaches its extreme values, the signal values will fit into (11) modified by the amplitude $A_{\text {int }}$ of the interferer. Two straight lines can be fitted into these extreme values, illustrated in Fig. 4. If now $A_{\text {int }}$ is known, $c_{1}$ and $c_{2}$ in (11) can be determined out of the fitted straight line. With them the interfering signal's frequency dependance is known. As there are two possible solutions for the fit, an uncertainty of a factor -1 remains.

To estimate $A_{\text {int }}$, again (5) is taken into account. As the interference component has a very high amplitude, it will determine the locations of extreme values of $s_{\mathrm{Rx}}(t)$. At the extrema, the signal value is a composition of $A_{\text {int }}$ and the sum of the target signals with time dependant instantaneous phases which is described as a random value $x$ oscillating around zero:

$$
s_{\mathrm{Rx}, \max }=A_{\mathrm{int}}+x .
$$

By calculating the mean value of the extrema's absolutes, $A_{\text {int }}$ can be determined, as the mean of $x$ will disappear:

$$
\frac{1}{N} \sum_{i=1}^{N} s_{\mathrm{Rx}, \max }=\frac{1}{N} \sum_{i=1}^{N} A_{\mathrm{int}}+\frac{1}{N} \sum_{i=1}^{N} x \approx A_{\mathrm{int}} .
$$

The information gained is used to reconstruct and remove the interfering signal $s_{\text {int }}(t)$ from the total received signal $s_{\mathrm{Rx}}(t)$ by calculating

$$
\begin{aligned}
s_{\text {int }}(t) & =A_{\text {int }} \cos \left(\int\left(\frac{d}{d t} \varphi_{\text {int }}(t)\right) d t\right) \\
& =A_{\text {int }} \cos \left(\frac{1}{2} c_{1} t^{2}+c_{2} t+\varphi_{0}\right) .
\end{aligned}
$$

With integration, an unkown constant $\varphi_{0}$ arises in the cosine representing the zero phase of the interfering signal. To get hold on this constant, two approaches are suggested. The first one will just estimate $\varphi_{0}$ in the time domain signal $s_{\mathrm{Rx}}(t)$. Taking a look at Fig. 1, at the cutting point of the two frequency ramps their difference is zero, so the phase is changing very slow here. That makes this point well suited for an estimation, the point in time is called $t_{\text {int }}$. Setting of (11) to zero and insertion in (14) leads to

$$
s_{\mathrm{Rx}}\left(t_{\text {int }}\right)=A_{\text {int }} \cos \left(\varphi_{0}-\frac{c_{2}^{2}}{2 c_{1}}\right)+x .
$$

Here, $x$ is the same as in (12). By assuming $\mathrm{x}$ was small, a starting point for an estimation of $\varphi_{0}$ is given. This value then is optimized, e.g. by using the knowledge on extrema's locations in $s_{\mathrm{Rx}}(t)$. Afterwards, the signal repair can be performed. This procedure is strongly dependant on the quality of determining $\varphi_{0}$. However, in the following another approach is presented not having this issue.

By applying an I-Q mixer, the estimation of $\varphi_{0}$ is redundant. The mixer provides sine and cosine component of the input signal $s_{\mathrm{Rx}}(t)$. When (5) is considered the I-component, the Q-component will be

$$
s_{\mathrm{Rx}, \mathrm{Q}}(t)=\sum_{i} A_{i} \sin \left(2 \pi f_{i} t+\varphi_{i}\right)+A_{\mathrm{int}} \sin \left(\varphi_{\mathrm{int}}(t)\right) .
$$

The derivative of the I-component is given analogous to (9):

$$
\frac{d}{d t} s_{\mathrm{Rx}, \mathrm{I}}(t) \approx-A_{\mathrm{int}} \sin \left(\varphi_{\mathrm{int}}(t)\right) \frac{d}{d t} \varphi_{\mathrm{int}}(t) .
$$

The values of $A_{\text {int }}$ and $\frac{d}{d t} \varphi_{\text {int }}(t)$ are estimated following the previous algorithm. Afterwards, (17) is divided by $\frac{d}{d t} \varphi_{\text {int }}(t)$ and added to (16). Doing so, the interference component is removed from the signal. As there were two possible solutions for the fit performed in Fig. 4, it has to be checked and corrected if necessary whether the correct sign for $\frac{d}{d t} \varphi_{\text {int }}(t)$ was chosen. 


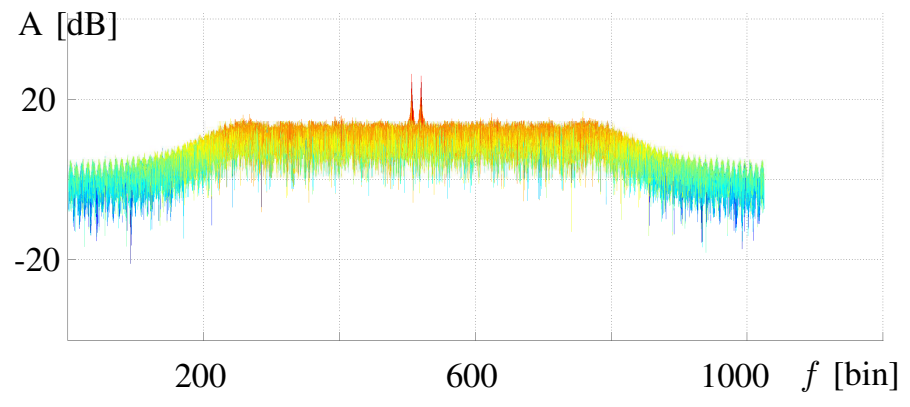

Fig. 5: Simulated 2D spectrum of 256 interfered chirp ramps. The chosen parameters were $B_{\mathrm{Rx}}=200 \mathrm{MHz}, f_{\mathrm{S}}=40 \mathrm{MHz}$, $T_{\mathrm{c}}=25.6 \mu \mathrm{s}$, and receiver bandwidth $B_{\mathrm{Rx}}=18 \mathrm{MHz}$ at a center frequency $f_{\mathrm{c}}=77.1 \mathrm{GHz}$.

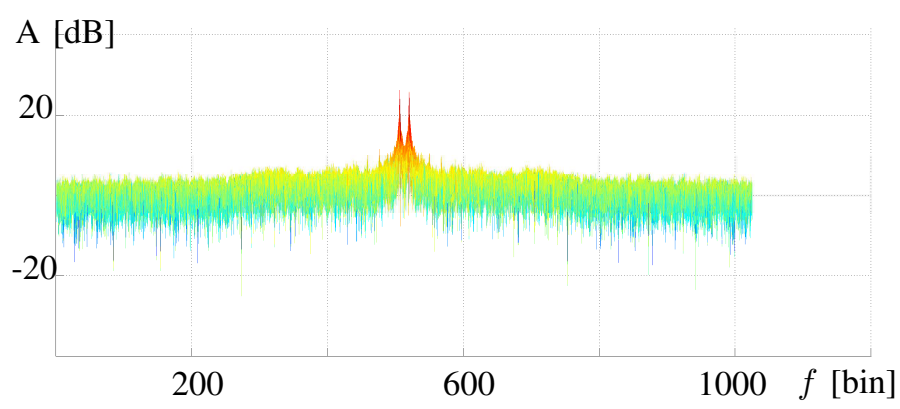

Fig. 6: Same spectrum as in Fig. 5, but with the interference repaired by the proposed method. Thereby, it was possible to decrease the noise floor by approximately $10 \mathrm{~dB}$.

\section{Simulation}

To show the method's functionality, simulations were performed repairing a signal with one interferer present. 256 chirp ramps were simulated with the parameters given in Fig. 5. An interferer using the same modulation scheme but a different sign for the frequency ramp slope was present at a random point in time for each chirp, a window function was not applied. Using the repair algorithm with estimation of $\varphi_{0}$, it was possible to suppress the interferer's influence as shown in Fig. 6.

\section{Discussion}

Finally, advantages and disadvantages of the proposed method compared to just replacing interfered samples by zeros are adressed.

Advantages are that no information is lost, as the signal is repaired, and that accuracy of the method is increasing with raising interference period, as parameter estimation is getting better with the number of available points. Blinding out interfered signal parts is getting worse the longer the interference lasts. Another advantage is, that not all corrupted samples have to be detected. With the parameters estimated, the interference period can be calculated. Additionally, knowledge on an input filter can be taken into consideration.

Disadvantageous at the procedure is the expected high calculation effort to receive enough accuracy with the estimation to still be effective.

\section{CONCLUSION}

A method for time domain interference mitigation was presented estimating amplitude and frequency behaviour of an interfering signal. This information is used to reconstruct and remove the interference component in time domain. Compared to just cutting out the signal part affected by interference, this method can avoid a loss of signal information. However, it requires higher calculation effort. Its effectivity increases with increasing duration of interference, as this makes parameter estimation more accurate. Future investigations will apply the method on a hardware system and determine the maximum possible SNR gain by optimization of the applied algorithms. As interference duration is considered a significant issue as well, its influence will also be taken into account.

\section{REFERENCES}

[1] D. Oprisan and H. Rohling, "Analysis of mutual interference between automotive radar systems," 2005.

[2] G. M. Brooker, "Mutual Interference of Millimeter-Wave Radar Systems," IEEE Transactions on Electromagnetic Compatibility, vol. 49, no. 1, pp. 170-181, February 2007.

[3] M. Goppelt, H.-L. Blöcher, and W. Menzel, "Automotive radar investigation of mutual interference mechanisms," Advances in Radio Science, vol. 8, pp. 55-60, 2010. [Online]. Available: http://www.advradio-sci.net/8/55/2010/

[4] M. Goppelt, H.-L. Blöcher, and W. Menzel, "Analytical investigation of mutual interference between automotive FMCW radar sensors," in Microwave Conference (GeMIC), 2011 German, March 2011, pp. 1-4.

[5] C. Fischer, M. Goppelt, H.-L. Blöcher, and J. Dickmann, "Minimizing interference in automotive radar using digital beamforming," Advances in Radio Science, vol. 9, pp. 45-48, 2011. [Online]. Available: http://www.adv-radio-sci.net/9/45/2011/

[6] L. Mu, T. Xiangqian, S. Ming, and Y. Jun, "Research on key tchnologies for collision avoidance automotive radar," in Intelligent Vehicles Symposium, 2009 IEEE, June 2009, pp. 233-236.

[7] V. Winkler, "Range doppler detection for automotive fmcw radars," in Microwave Conference, 2007. European, Oct 2007, pp. 1445-1448. 